\title{
Nano interface potential influences in CdTe quantum dots and biolabeling
}

\author{
S. Kanagasubbulakshmi ${ }^{1} \cdot \mathrm{K}_{\text {. Kadirvelu }}{ }^{1}$
}

Received: 4 August 2017 / Accepted: 12 April 2018 / Published online: 4 May 2018

๑) Springer-Verlag GmbH Germany, part of Springer Nature 2018

\begin{abstract}
Nano interface influences in physiochemical properties of quantum dots (QDs) are the challenging approach to tailor its surface functionalities. In this study, a set of polar and non-polar solvents were selected to analyze the influences in solventbased dynamic radius and surface potential of QDs. From the nano interface chemistry of polar and non-polar solvents, an appropriate mechanism of precipitation and hydrophobic ligand exchange strategy were elucidated by correlating Henry's equation. Further, the in vitro cytotoxic potential and antimicrobial activity of QDs were assessed to perform biolabeling. From the observations, an appropriate dosage of QDs was fixed to label the animal ((RAW 264.7 cell lines) and bacterial cells (Escherichia coli) for effective cell attachment. Biolabeling was achieved by tailoring nano interface chemistry of QDs without additional support of biomolecules. Bacterial cell wall-based interaction of QDs was evaluated using SEM and EDAX analysis. Thus, provided clear insights into the nano interface chemistry in the development of highly photostable QDs will be helpful in biomedical applications.
\end{abstract}

Keywords Quantum dots $\cdot$ CdTe $\cdot$ Nano interface $\cdot$ Cytotoxicity $\cdot$ Biolabeling

\section{Introduction}

Over the past decade, the surface chemistry of nanomaterials has been considered as an active scientific research and a powerful toolkit for materials engineering. Factors behind the chemical bonding and electronic structure at nanoscale interfaces have improved tremendously with the help of new experimental and computational techniques capable of shedding light upon this challenging class of objectives (Boles et al. 2016). The tremendous growth in nanotechnology leads to the engineered QDs that can be used in different fields of biomedical and pharmaceutical sciences, like biosensing, antibiotics, imaging and drug delivery (Vergaro et al. 2011). Thus, interfacial potential formed on the interaction of QDs with biomolecular surfaces becomes a very

Electronic supplementary material The online version of this article (https://doi.org/10.1007/s13204-018-0774-0) contains supplementary material, which is available to authorized users.

K. Kadirvelu

kadirvelukrishna@yahoo.com

1 DRDO-BU Center for Life Sciences, Bharathiar University Campus, Coimbatore, Tamil Nadu 641046, India important factor to study, prior to its use for any biological applications (Arakha et al. 2015).

In the applications of QDs, controlling the size, morphology and surface ligands is crucial because it can lead to a significant change in their optical properties Especially, fluorescence quenching of QDs is one of the most interesting photochemical phenomena that can be applied in many areas including molecular sensing and imaging probes (Noh et al. 2010). As such, establishing the link between QDs surface structure and optical properties remains a crucial open question in the field. Surface passivation upon ligand binding is common but not universal: some ligands introduce new miDgap electronic states and increase the rate of non-radiative relaxation (Wang et al. 2016).

Solvent molecules can alter the surface charge transfer process by perturbing the dielectric layer or through the dynamic configuration rearrangements (electronic and conformational) of the solvent molecules at the nanomaterial surface during charge transfer process. To dissolve QDs into solvents with different polarities, it is typically necessary to alter the ligand coverage or swap ligands entirely, convolving solvent effects with ligand effects. This makes it difficult to isolate the effect of the solvent alone (Ellis et al. 2015). The relationship between zeta potential, electrophoretic mobility 
and ambient conditions surrounding the QDs remains a largely unexplored area which will require researchers from many scientific disciplines to answer and this was taken as a challenge to reveal the solvent-specific influences by considering its physiochemical properties. The dielectric constant is a physical property, which is influenced by interatomic and intermolecular attractions. Henry's Equation gives the relationship between solvent properties, Zeta and Electrophoretic mobility of QDs (Oszwałdowski et al. 2013).

The zeta potential is defined as the electrical potential between the inner Helmholtz layer near a particle's surface and the bulk liquid in which a particle is suspended and this is a parameter that represents the charge of a particle (DeBlois et al. 1977). The zeta potential is particularly relevant in the transition zone where the system experiences continuous and large organizational changes in small temperature ranges (Sierra et al. 2016). Recent reports confirm the zeta potential and electrophoretic mobility mainly depends on the hydrodynamic radius $\left(R_{\mathrm{H}}\right)$ of the QDs created by solvents (Pyell 2010). As reported in solvents effect on zeta potential in an interface medium (Wu et al. 2014), it is essential to explore the interface properties of nanosystem by considering solvents physiochemical properties to explore the mechanism.

Much progress has recently been made in using semiconductor nanocrystals as fluorescent labels for biological samples. These QDs have advantages over traditional fluorescent probes due to their broad absorption, narrow emission spectra, and resistance to photobleaching. Unlike mammalian cells, bacteria do not endocytose, and internalization of QDs by prokaryotes needs target specific functional groups for labeling. However, the limited information about interactions of QDs and their bioconjugates/labeling with microorganisms confines the microbiological applications of QDs; such internal labeling would be desirable in many applications (Kloepfer et al. 2005). Generally, the targetspecific functional groups and cell wall treatments are followed to enhance the efficient attachment of QDs which make the steps complicated. Effective attachment of QDs could alter the microbial morphology which makes QDs not suitable for biolabeling. Surface oxidation was found to be the main reason for toxicity which alters the QDs functional properties. The proper capping and surface passivation could prevent oxidation as well as leaching of Cadmium ions into the biomatrix (Li et al. 2004; Wang et al. 2004; Schneider et al. 2009).

This study was an attempt to prove that solvents can able to modulate the local electrochemical environment by directing the redistribution of interface charges. Hydrodynamic radius $\left(R_{\mathrm{H}}\right)$ can also decide the QDs stability, aggregation, photoluminescence intensity, zeta potential, electrophoretic mobility and other related parameters. Toxicity assay (3-(4,5-dimethylthiazol-2-yl)-2,5-diphenyl tetrazoliumbromide-MTT) and antimicrobial activity of QDs were performed to understand the in vitro toxic potential of QDs and fixed dosage was used to label animal cells and Escherichia coli cells. On the basis of effective cell labeling by QDs, the distinct charge transfer between bio and nano interface chemistry is proposed for biomedical applications.

\section{Experimental section}

\section{Materials required}

Cadmium oxide (CdO), Octadecene (ODE), Oleic acid(OA), Tellurium(Te) and MPA (3-mercaptopropionic acid) Analar grade(AR) were obtained from Sigma-Aldrich and acetone, ethanol, methanol, chloroform and toluene were obtained from Merck India Pvt Ltd. Double distilled water was used for phosphate-buffered saline (PBS) preparation.

\section{Synthesis of CdTe QDs}

CdTe QDs were synthesized using reflux condensation method with minor modifications as reported elsewhere (Chen et al. 2009). Briefly, Oleic acid and Octadecene mixture was used to prepare Cadmium precursor at $180{ }^{\circ} \mathrm{C}$. Octadecene was used to prepare Tellurium precursor. The equimolar ratio was maintained to form a strong core of CdTe QDs. Once the monomers of precursors are ready, the swift hot injection was followed to allow the nucleation at $120^{\circ} \mathrm{C}$. Once the reaction has completed, the core QDs were suspended in toluene. Precipitation efficiency of commonly used solvents (acetone, ethanol and methanol) was analyzed by fixing the same volume ratios of QDs and solvents [1.5 mL solvent $+1.5 \mathrm{~mL}$ QDs $(1 \mu \mathrm{g} / \mu \mathrm{L})$ mixture]. The fluorescence characteristics of QDs were investigated spectrofluorimetrically by dissolving the obtained pellet (from the precipitation) in $3 \mathrm{~mL}$ of toluene and chloroform. Volume of solvents and concentration of QDs were maintained same in all interface chemistry analysis to evaluate appropriate factors that affect QDs properties.

\section{Hydrophilic ligand exchange}

As synthesized CdTe core QDs have replaceable ligands on QDs surface (Murray et al. 1993). Phase-separated QDs were taken in chloroform and toluene contained vial for ligand exchange process with MPA. A fixed concentration and volume of MPA were added to the $1 \mathrm{ml}$ of QDs solution and vortexed for $15 \mathrm{~min}$. As separated two layers were centrifuged and washed with ethanol, thus obtained pellet was collected and redispersed in a pH 7 PBS for further studies (Uyeda et al. 2005). 


\section{In vitro cytotoxic activity of M-CdTe QDs}

The cytotoxicity of the M-CdTe QDs was evaluated on RAW 264.7 cell lines by MTT assay. The RAW 264.7 cells were obtained from the National Centre for Cell Science (NCCS), Pune, India. The cells were maintained in Dulbecco's Modified Eagle's Medium (DMEM) supplemented with $10 \%$ fetal bovine serum (FBS), penicillin $(100 \mu \mathrm{g} /$ $\mathrm{mL})$ and streptomycin $(100 \mu \mathrm{g} / \mathrm{mL})$ under humidified atmospheric chamber at $5 \% \mathrm{CO}_{2}$ and $37^{\circ} \mathrm{C}$. The cells were sub-cultured in $75 \mathrm{~cm}^{2}$ culture flasks; media were replaced at every alternative day and confluent cells were used for the experiment. Approximately, $1 \times 10^{4}$ cells/well were seeded in 96-well plate and allowed to adhere for $6 \mathrm{~h}$. Following, cells were treated with different concentrations of M-CdTe QDs in DMEM devoid of FBS for $24 \mathrm{~h}$. The cells not exposed to M-CdTe QDs were considered as a control. Following, the MTT assay was performed as per the procedure of (Venkataramana et al. 2014). The optical density of formazan crystals was determined at $570 \mathrm{~nm}$ under multimode plate reader (Synergy H1, BioTek, USA) and results were expressed with respect to the control (100\%).

\section{In vitro imaging of animal cells with M-CdTe QDs}

The RAW 264.7 cells were seeded in 24-well cell culture plate and allowed to adhere for $6 \mathrm{~h}$. The cells were exposed with $\mathrm{M}-\mathrm{CdTe} \mathrm{QDs}$ at a dosage below $\mathrm{IC}_{50}$ value in $\mathrm{DMEM}$ devoid of FBS and incubated for $15 \mathrm{~min}$ at $37{ }^{\circ} \mathrm{C}$ under dark. The cells were washed with Dulbecco's phosphate buffered saline (D-PBS) and images were captured under red fluorescent protein (RFP) using EVOS FLC inverted fluorescent microscope (Life Technologies, USA).

\section{In vitro antimicrobial activity of M-CdTe QDs}

The antimicrobial activity of M-CdTe QDs was assessed on $E$. coli by micro-well dilution method. The bacterial culture was grown for overnight in Luria-Bertani (LB) broth and the cell count was adjusted to $1 \times 10^{6} \mathrm{cfu} / \mathrm{mL}$. Different concentrations of M-CdTe QDs and $10 \mu \mathrm{L}$ of bacterial suspension were added to a 96-well microtiter plate and total volume was adjusted to $100 \mu \mathrm{L}$ with LB broth. The wells not exposed to M-CdTe QDs were considered as a control and plate was incubated for $24 \mathrm{~h}$ at $37^{\circ} \mathrm{C}$. The optical density was measured at $620 \mathrm{~nm}$ under multimode plate reader (Synergy H1, BioTek, USA) and the minimum concentration of M-CdTe QDs with no evident growth was defined as minimum inhibitory concentration (MIC).

\section{Biolabeling of $E$. coli}

A volume of $1 \mathrm{~mL}$ bacterial suspension $\left(1 \times 10^{6} \mathrm{cfu} / \mathrm{mL}\right)$ was stained with a lower MIC of M-CdTe QDs for 15 min under dark. The bacterial cells unstained with M-CdTe QDs were considered as a control. The cells were washed with PBS at $3000 \mathrm{rpm}$ for $5 \mathrm{~min}$ and cells were fixed onto glass slides ( $\mathrm{Li}$ et al. 2004). The cells were observed under red fluorescent protein (RFP) using EVOS FLC inverted fluorescent microscope (Life Technologies, USA).

\section{Characterization}

Both the hydrophobic and hydrophilic QDs were characterized to confirm the photophysical properties. Absorbance spectra were recorded using a UV-Vis-NIR spectrophotometer (SHIMADZU 3600 model). Emission spectra were recorded using a Spectrofluorometer (Horiba Jobin-Yvon Fluromax 4). Hydrodynamic radius, electrophoretic mobility and Zeta potential were analyzed using ZETASIZER MALVERN MAKE. Surface morphology, the size of QDs and SAED pattern were examined using HR-TEM (JOEL JEM 2100 at $200 \mathrm{kV}$ ). To identify bonding patterns, the infrared spectra were taken using FT-IR (SHIMADZU 8400S model). XRD was taken using PANalytical X'Pert Pro instrument. SEM and EDAX (FEI-QUANTA 200) studies were performed to understand the attachment of QDs on the microbial cell wall. Fluorescence imaging was obtained using EVOS FLC inverted fluorescent microscope (Life Technologies).

\section{Results and discussion}

\section{Nano interface chemistry}

The commonly used solvents for precipitation of QDs from the organic mixture were ethanol, acetone and methanol. Once the precipitation and phase separation of QDs from the organic mixture have been done, the QDs were dispersed in non-polar solvents such as toluene and chloroform to analyze the ligand exchange efficacy of the suitable medium. Depending on the solvents parameters and electrical double layer (EDL), the surface exchangeable ligands of QDs get alter and thus affect the hydrophilic ligand exchange. As per Henry's equation solvents, physiochemical properties (Table 1) have a specific role that corresponds to surface interface potential changes.

\section{Hydrodynamic radius $\left(\boldsymbol{R}_{\mathrm{H}}\right)$}

The smallest hydrodynamic radius $(3.9 \mathrm{~nm})$ was ascribed to single QDs, whereas the bigger ones $(28.49 \mathrm{~nm})$

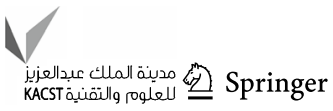


Table 1 Physical properties of solvents used in the analysis (Sigma-Aldrich)

\begin{tabular}{llllll}
\hline S. no. & Solvents & Density g/ml & Viscosity $(\mathrm{cp})$ & Refractive index & $\begin{array}{l}\text { Dielectric } \\
\text { constant }\end{array}$ \\
\hline 1. & Acetone & 0.791 & 0.32 & 1.359 & 20.7 \\
2. & Ethanol & 0.816 & 1.10 & 1.363 & 24.55 \\
3. & Methanol & 0.791 & 0.55 & 1.329 & 32.60 \\
4. & Toluene & 0.865 & 0.59 & 1.496 & 2.40 \\
5. & Chloroform & 1.492 & 0.58 & 1.446 & 4.81 \\
\hline
\end{tabular}

corresponded to the aggregates of QDs. Recent works suggest that the $R_{\mathrm{H}}$ of QDs could be considerably larger than their measured TEM 'dry' radii, but the reported TEM values do not represent the true physical sizes of organic-coated QDs. The reason is that organic materials (such as OA, MPA and other polar or non-polar solvents) are not electron dense enough for TEM visualization on the nanometer scale (Gao et al. 2004). It is also observed that solvent's physiochemical properties cause severe variation in the $R_{\mathrm{H}}$ of QDs (Table 2). The influences of solvents were found as mentioned below:

\section{Methanol > Acetone > Ethanol.}

Dielectric constant plays a crucial role to sustain the monodispersity of particles; the cation concentration of methanol is very high when we compared to other two solvents, which will not permit agglomeration of the particles (Jiang and Muscat 2013). This phenomenon could be evoked as an explanation for the solvent-based influences in agglomeration and $R_{\mathrm{H}}$ of the QDs.

\section{Zeta potential}

The influences of solvents in zeta potential were found to be in the same order of magnitude as correspond to $R_{\mathrm{H}}$ of QDs. Methanol has high polarity than other two solvents (ethanol and acetone); the zeta potential moved to negative with increasing solvent polarity. The reason is due to the accumulation of more cations at the QDs ligands/solvent interface as a result of the electron-donating property of the hydroxyl substituent, which makes a net negative charge and makes the QDs highly stable.
Based on the physical parameters as explained previously, the potential medium for stable ligand exchange was found to be chloroform which gave high zeta potential $(-32.9 \mathrm{eV})$ and electrophoretic mobility $(2.58 \mathrm{~cm} / \mathrm{Vs})$ than toluene exchange medium. It can be concluded that reduction of the surface charges on QDs may be due to strong binding of solvents with QDs (Smith et al. 2006). The zeta potential is changing according to the changes in the solution, which means that by zeta-potential measurements it is possible to follow the changes, which take place in the solution (Pattekari et al. 2011). When the potential is small, attractive forces may exceed this repulsion and the dispersion may break and flocculate. Colloids with high zeta potential (negative or positive) are electrically stabilized while colloids with low zeta potentials tend to coagulate or flocculate (Zheng et al. 2011). From this observation, it can be concluded that an efficient interface could provide the non-aggregated QDs with less toxicity (Wuister et al. 2004).

\section{Quenching of photoluminescence}

A physisorption of solvents with different interaction strength on dot surface states can be invoked as an explanation for photoluminescence (PL) quenching. The polarity of solvents influences the concentration of QDs extraction, particle sizes, monodispersity, surface passivation, and surface states. In the case of acetone and ethanol, the polar nature and physical properties (Table 1) lead to strong bonding with (OA) ligands present on the QDs surface. The complex formation leads to aggregation of QDs and quenches the PL (Fig. 1). In the case of methanol, the dielectric layer around
Table 2 Zeta potential, electrophoretic mobility and hydrodynamic radius disparity by solvent influences

\begin{tabular}{llllc}
\hline S. no. & $\begin{array}{l}\text { Precipitation + ligand } \\
\text { exchange medium }\end{array}$ & $\begin{array}{l}\text { Zeta potential } \\
(-\mathrm{mV})\end{array}$ & $\begin{array}{l}\text { Electrophoretic mobil- } \\
\text { ity }(\mathrm{cm} / \mathrm{Vs})\end{array}$ & $\begin{array}{l}\text { Hydrody- } \\
\text { namic radius } \\
(\mathrm{nm})\end{array}$ \\
\hline 1. & Acetone + Toluene & 14.6 & 0.961 & 26.83 \\
2. & Ethanol + Toluene & 11.2 & 0.742 & 28.49 \\
3. & Methanol + Toluene & 22.9 & 1.51 & 8.34 \\
4. & Acetone + Chloroform & 14.7 & 0.969 & 6.04 \\
5. & Ethanol + Chloroform & 12 & 0.795 & 6.87 \\
6. & Methanol + Chloroform & 32.9 & 2.58 & 3.9 \\
\hline
\end{tabular}

مدينة الملك عبدالعزيز KACSTT 


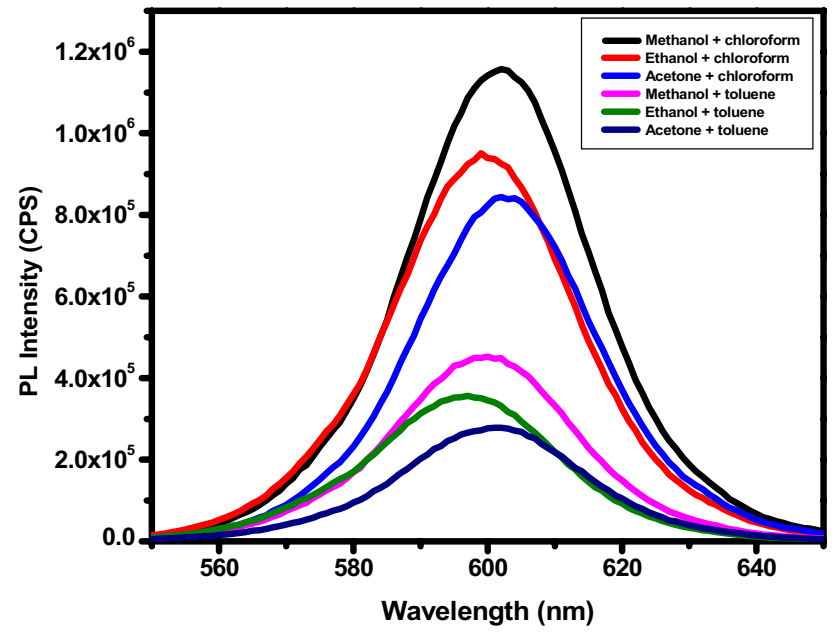

Fig. 1 Photoluminescence spectra of CdTe QDs dispersed in nonpolar solvents based on quenching efficiency of polar solvents

the QDs which depends on dielectric constant gives less aggregation of QDs and repels each other and gives highly monodispersed QDs for hydrophilic ligand exchange. The dielectric layer around the QDs by ethanol and acetone leads to the charge trapping of electrons, thus affecting the PL intensity of QDs which further leads to less stability. On other hand, the non-polar nature of chloroform and toluene have not lead to complex formation and makes the surface exchangeable ligands to available freely for further ligand exchange process (Wu et al. 2003). Density is the main parameter for efficient ligand exchange as it decides the diffusion rate of MPA in the solvent. As the density of a solvent increases, the rate of diffusion decreases, thus providing a stable surface for ligand exchange. Slow diffusion rate also provides the better passivation of the QDs surface and uniform dispersion of Thiol and Carboxyl groups, which enhances the stability of QDs. From Fig. 1, the chloroform was found to be potential hydrophilic ligand exchange medium; thus, it has a high density (1.492) than toluene. From this observation, the methanol was found to be efficient precipitator and chloroform was found to be potential ligand exchange medium.

\section{Characterization of M-CdTe QDs}

\section{Optical properties}

After optimization of solvent-based parameters, the highly stable QDs were synthesized with sufficient knowledge in the clear insight of QDs surface properties. The absorbance and emission spectrum of synthesized CdTe QDs are shown in Fig. 2a, b. The bare CdTe QDs absorbance and emission were increased and shifted towards higher wavelength when it is capped by MPA. The excitation spectrum of M-CdTe QDs is illustrated in Fig. S2 that shows the peaks in the range of $450-500 \mathrm{~nm}$. The surface chemistry alteration leads to the energetic positions on the top of the valence bands in CdTe QDs. The energy difference between the top of the valence bands of bulk CdTe is more than $0.5 \mathrm{eV}$. For CdTe, the valence band is situated at higher energies with respect to the redox level of most thiols, thus inhibiting hole trapping and maintaining high luminescence efficiency. If the hole is trapped in a thiol molecule, radiative recombination
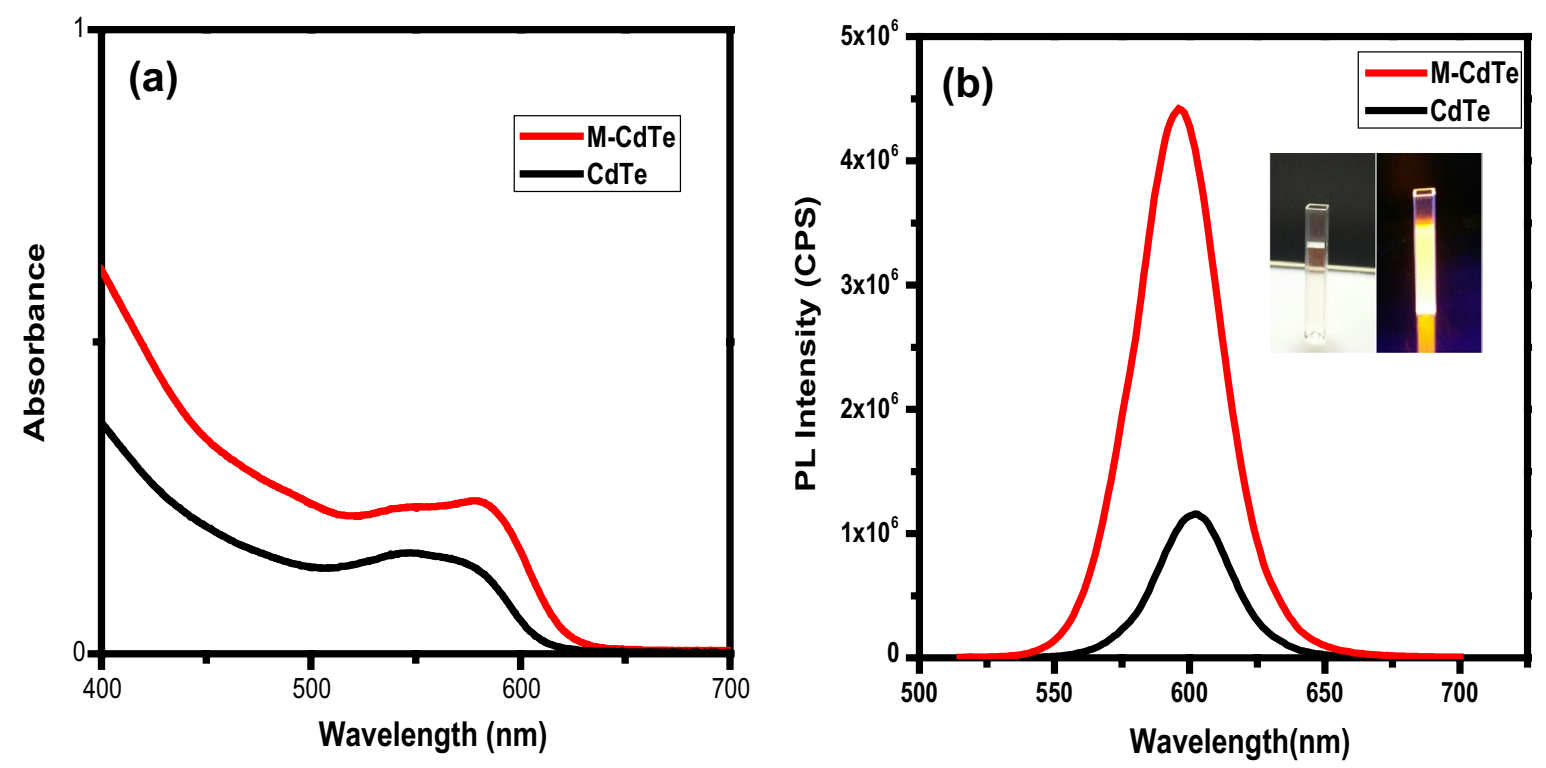

Fig. 2 Absorbance and emission spectra of CdTe and M-CdTe QDs [inset—QDs under UV illumination (365 nm)] 
of the exciton is not possible resulting in a strongly reduced quantum efficiency (QE) (Zhang et al. 2003).

\section{Morphological observation}

The size and morphology of QDs are shown in Fig. 3a, b, at different magnification showing that the QDs are highly monodispersed and uniform in size under HR-TEM observation. The size of the QDs was found to be $2.5 \mathrm{~nm}$ in average since the MPA surface modification does not lead to the agglomeration of QDs. The well-resolved lattice fringes from selected area electron diffraction (SAED) analysis reveal high crystalline nature of the QDs. Lattice parameters (311), (220), and (111) of CdTe QDs (Fig. 3 inset) illustrate that M-CdTe QDs have cubic zinc blende structure (Gao et al. 1998). (JCPS PDF 65-1046) SAED pattern of M-CdTe QDs was compared with XRD pattern (Fig. S1), the result was well correlated with the hkl values (311), (220), and (111) and cubic zinc blende structure of QDs were confirmed.

\section{Infrared bonding patterns}

Functional groups present on the surface of QDs were analyzed by FT-IR spectrum shown in Fig. 4. Here, the main concept of making hydrophobic QDs into hydrophilic QDs was achieved by the functional groups present on the surface of QDs mainly by - $\mathrm{COOH}$ which is an electron acceptor and gives a negative charge on the surface. Stretching at $3408.2,2927.94 \mathrm{~cm}^{-1}$ for toluene medium and 3417.86, $3022.45 \mathrm{~cm}^{-1}$ for chloroform medium corresponds to $\mathrm{H}$ bonded $-\mathrm{OH}$ vibration on the surface which denotes the

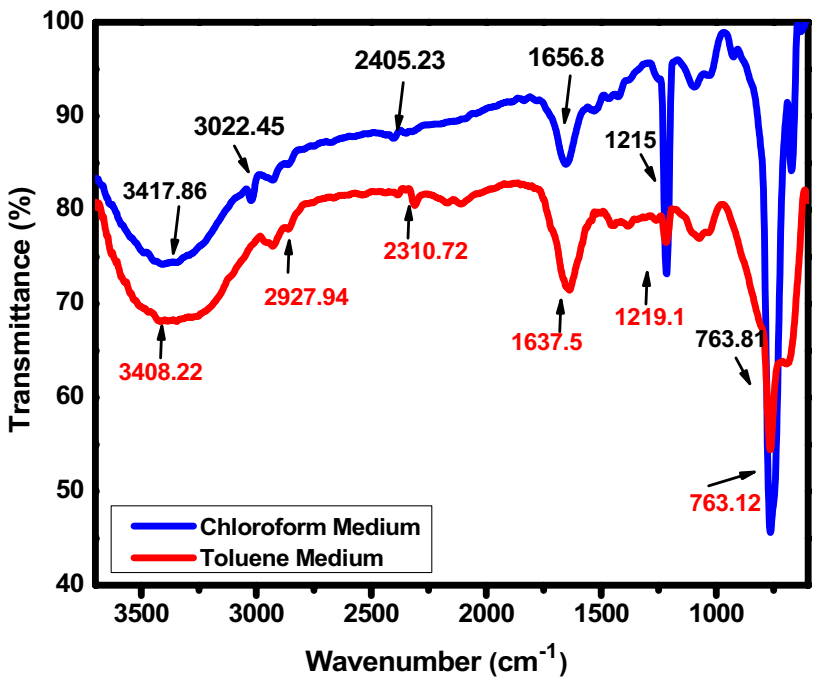

Fig. 4 FT-IR spectra of ligand exchange medium influences in M-CdTe QDs

covalent bond formation between MPA and CdTe QDs. The stretching vibration of the thiol group $2310.72 \mathrm{~cm}^{-1}$ for toluene and $2405.23 \mathrm{~cm}^{-1}$ for chloroform was not formed which clearly proves thiol-QDs covalent linkage. Stretching at 1656.8 and $1637.5 \mathrm{~cm}^{-1}$ denotes the $\mathrm{C}=\mathrm{O}$ vibration on the QDs and the significant shift in the asymmetric stretching vibration of the carboxyl groups were observed at 1215 and $1219.1 \mathrm{~cm}^{-1}$. As reported in Jhonsi and Renganathan (2010), due to the formation of covalent bonds between thiol as well as carboxyls and the $\mathrm{Cd}^{2+}$ ions of the surface of QDs, the peaks of $-\mathrm{SH}$ and $-\mathrm{COOH}$ groups on the surface of QDs were formed. This indicates that the thiol group interacted
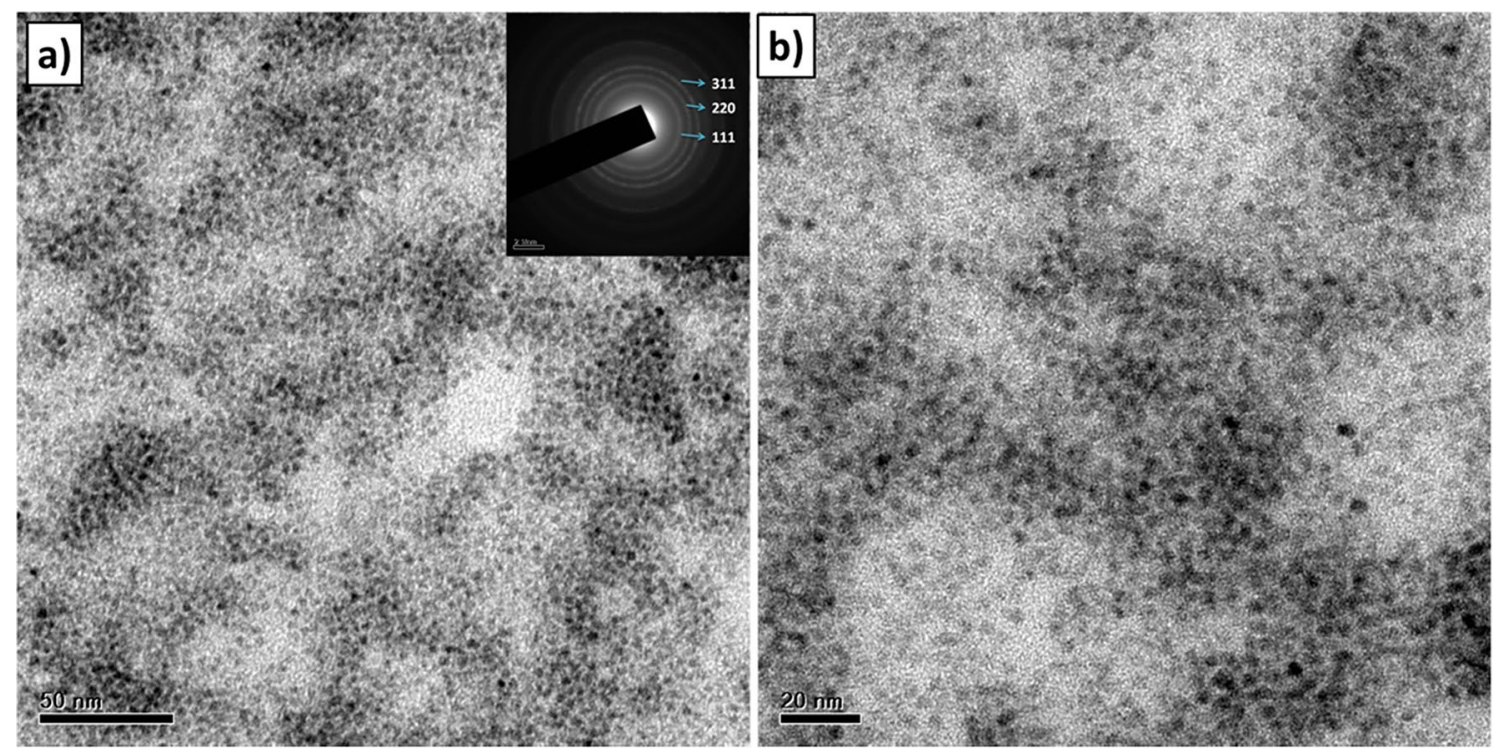

Fig. 3 HR-TEM images of M-CdTe QDs under different magnification (inset shows the SAED pattern of M-CdTe QDs) 
with the surface of $\mathrm{CdTe}$ and leaves - $\mathrm{COOH}$ group as functional head (Gaponik and Rogach 2010). In a fingerprint region stretching vibration at $763.12 \mathrm{~cm}^{-1}$ for toluene and $763.81 \mathrm{~cm}^{-1}$ for chloroform denotes the deformation of $\mathrm{C}-\mathrm{H}$ group present in MPA. When compared to chloroform, the toluene does not give strong deformation of $\mathrm{C}-\mathrm{H}$ group and proves the strong medium for ligand exchange is chloroform.

\section{In vitro cytotoxic potential of M-CdTe QDs quantum dots}

In consideration of the possible biomedical application and to prove biocompatibility of the synthesized M-CdTe QDs, the toxicity assay was performed. MTT assay was performed with various concentrations of QDs. The dose-dependent relative decrease in the viability of cells was observed with increasing concentration of QDs (Fig. 5). Commercially available doxorubicin drug was used as a positive control (Lüpertz et al. 2010) and the $\mathrm{IC}_{50}$ value was found to be $2.48 \mu \mathrm{g} / \mathrm{mL}$ (Fig. 6a). The cell viability was calculated by setting the viability of the control cells as $100 \%$. $\mathrm{IC}_{50}$ value was calculated as $150 \pm 3.6 \mu \mathrm{g} / \mathrm{mL}$ and $\mathrm{IC}_{90}$ was $225 \mu \mathrm{g} / \mathrm{mL}$ (Fig. 6b). When compared to previous studies (Schneider et al. 2009), QDs oxidation was well prevented by MPA passivation, thus reducing the toxicity of Cadmium (Cd) ions and preventing leaching from the core.

Fig. 5 In vitro cell line toxicity study a control RAW 264.7 cells, b positive control (Doxorubicin), $\mathbf{c}, \mathbf{d} \mathrm{IC}_{50}$ and $\mathrm{IC}_{90}$ infection by M-CdTe QDs
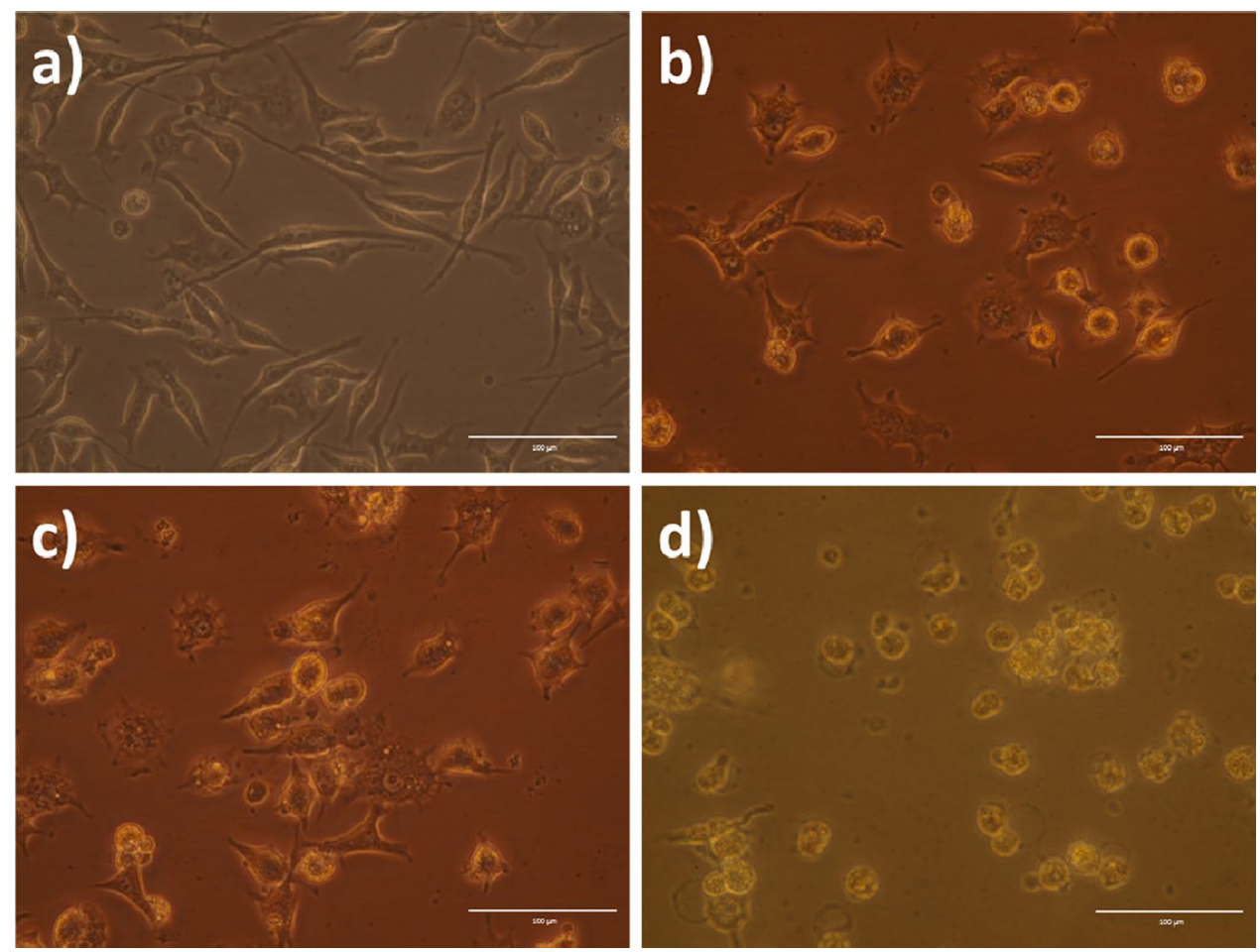
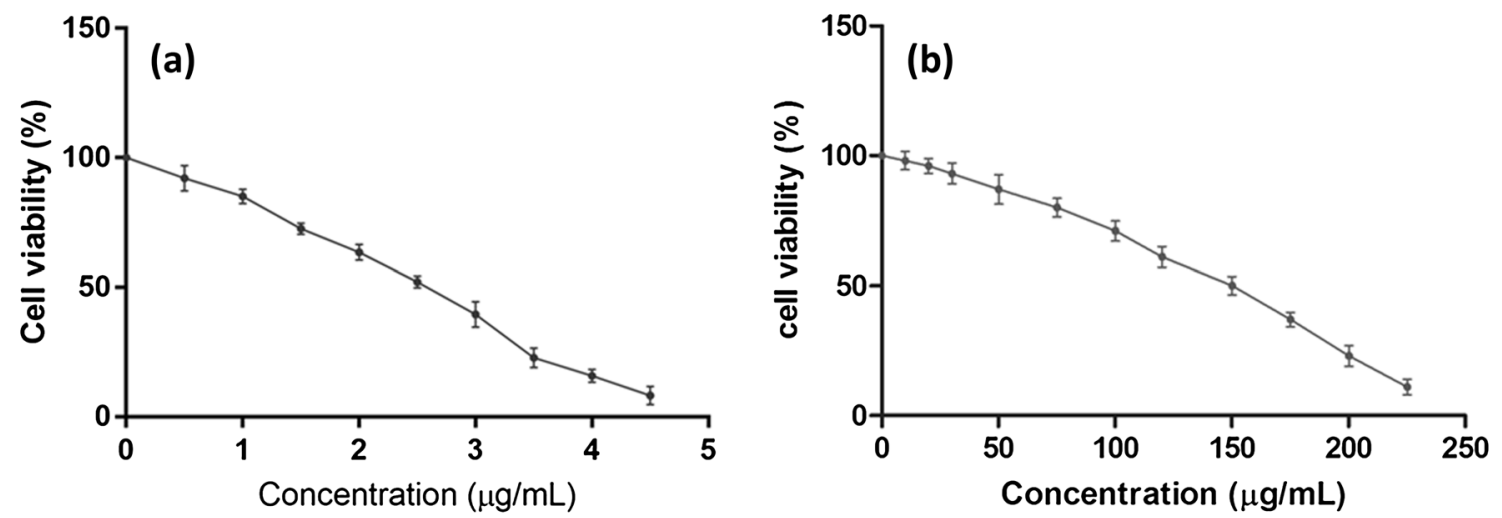

Fig. 6 Cytotoxicity of M-CdTe QDs on RAW 264.7 cells at various concentrations 


\section{In vitro imaging potential}

To investigate the fluorescence capability of QDs into the biomatrix, the RAW 264.7 cell lines were used for in vitro imaging. The prior knowledge about cytotoxic potential was considered while fixing the dosage for bioimaging. Figure 7 proves that a very small amount $(0.1 \mu \mathrm{g} / \mathrm{mL})$ of M-CdTe QDs have the capability of interacting with the cells at cytoplasmic level. When compared to previous studies (Zhang et al. 2013), very less dosage of QDs was used to visualize the cells, which is due to an enhancement of quantum efficiency by better surface passivation. Bioimaging of cell lines without surface capping (MPA) is shown in Fig. S3, which depicts that the hydrophobicity of CdTe QDs does not allow the QDs to penetrate into the cell lines. Bioprobing of QDs with additional biomolecules (antibody, aptamer and protein) for target-based binding would be useful in many biomedical applications.

\section{In vitro antimicrobial activity of M-CdTe QDs}

Antimicrobial activity of M-CdTe QDs was assessed on $E$. coli cells and expressed in the equal time interval $(6,12$ and $24 \mathrm{H}-$ Fig. 8). From the optical density of bacterial cells, the MIC required to suppress the bacterial growth was obtained and it was found to be less when compared to the previous article (Schneider et al. 2009; Du et al. 2018). Error bars in Fig. 8 have indicated that the triplicate results of antimicrobial activity have not shown any significant deviations from the primary observation. As fixed dosage from the antibacterial activity was used to explore the charge transfer-based biolabeling of E. coli cells.

\section{In vitro biolabeling of bacteria}

This study was an attempt to prove that the labeling is possible without endocytosis of bacterial cells by charge transfer between the QDs ligand and bacterial cell wall. A variety of methods for bacterial immobilization is based on antibody-antigen interactions, electrostatic and the physical

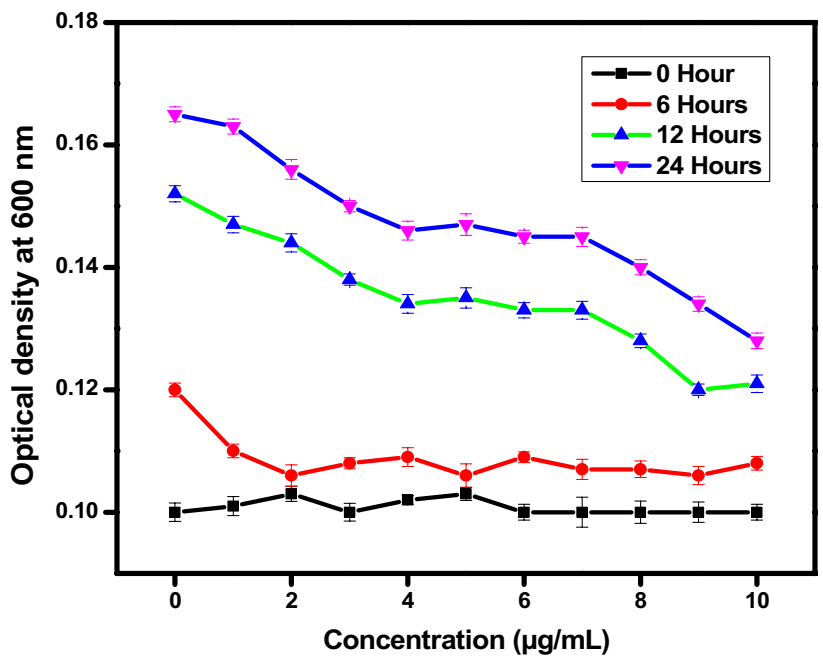

Fig. 8 Growth inhibition of E. coli by freshly prepared M-CdTe QDs at equal time interval

entrapment of bacteria (Michalet et al. 2005). Here, carboxyl-terminated QDs were further used to tag the bacteria cells. Since $E$. coli is a Gram-negative bacteria, the cell wall is more protective by its lipid bilayer; it is difficult to penetrate the cell wall by QDs. In this study, specific conjugation of QDs with bacterial cell wall was observed under fluorescence microscopy. Tagged bacterial cells agglomeration was glowing as a lane of light which does not affect the fluorescence of QDs. The net negative charge of bacterial cell wall and the charge neutralization on the surface of QDs by PBS buffer enhance the effective charge transfer mechanism between QDs and outer membrane of bacteria. As shown in Fig. 9, the step by step QDs coverage on the surface of the bacterial cell and cell agglomeration does not lead to fluorescence quenching due to the highly negative net surface charge. Magnified images of bacterial cell wall labeling are illustrated in Fig. S4 to prove the cell wall-based interaction of QDs.

SEM with EDX (Fig. 10) observation was utilized to identify the morphology and membrane integrity of the
Fig. 7 Bioimaging of RAW 264.7 cell lines: a M-CdTe QDs uptake by the cells under transmittance light image and b M-CdTe QDs uptake by the cells under RFP filter
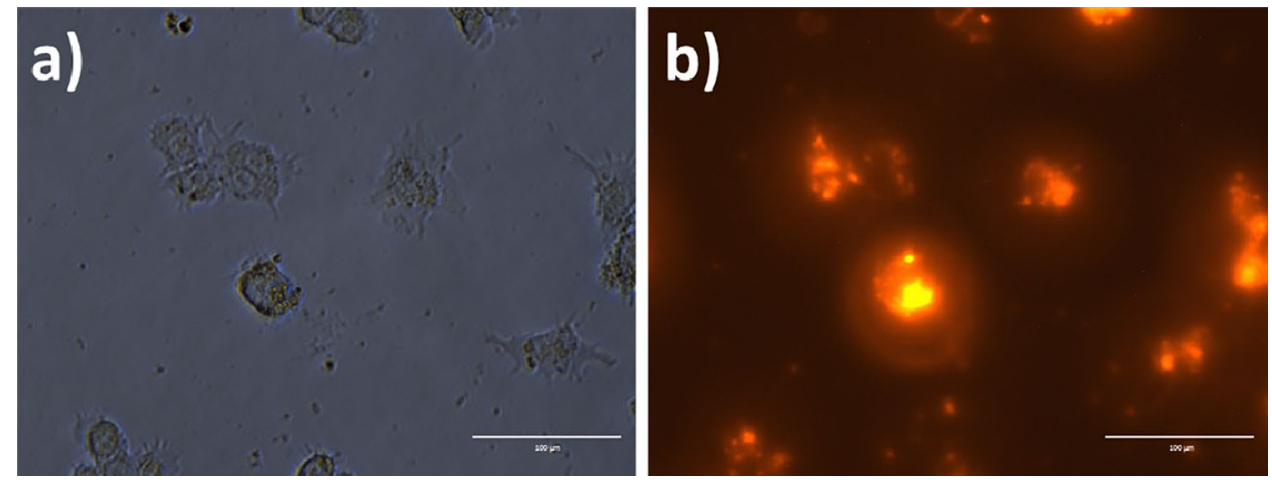
Fig. 9 Biolabeling of E. coli by M-CdTe QDs: a, b under transmittance light images of bacterial cells corresponding to fluorescence images. c, d QDs labeled $E$. coli cells under RFP filter
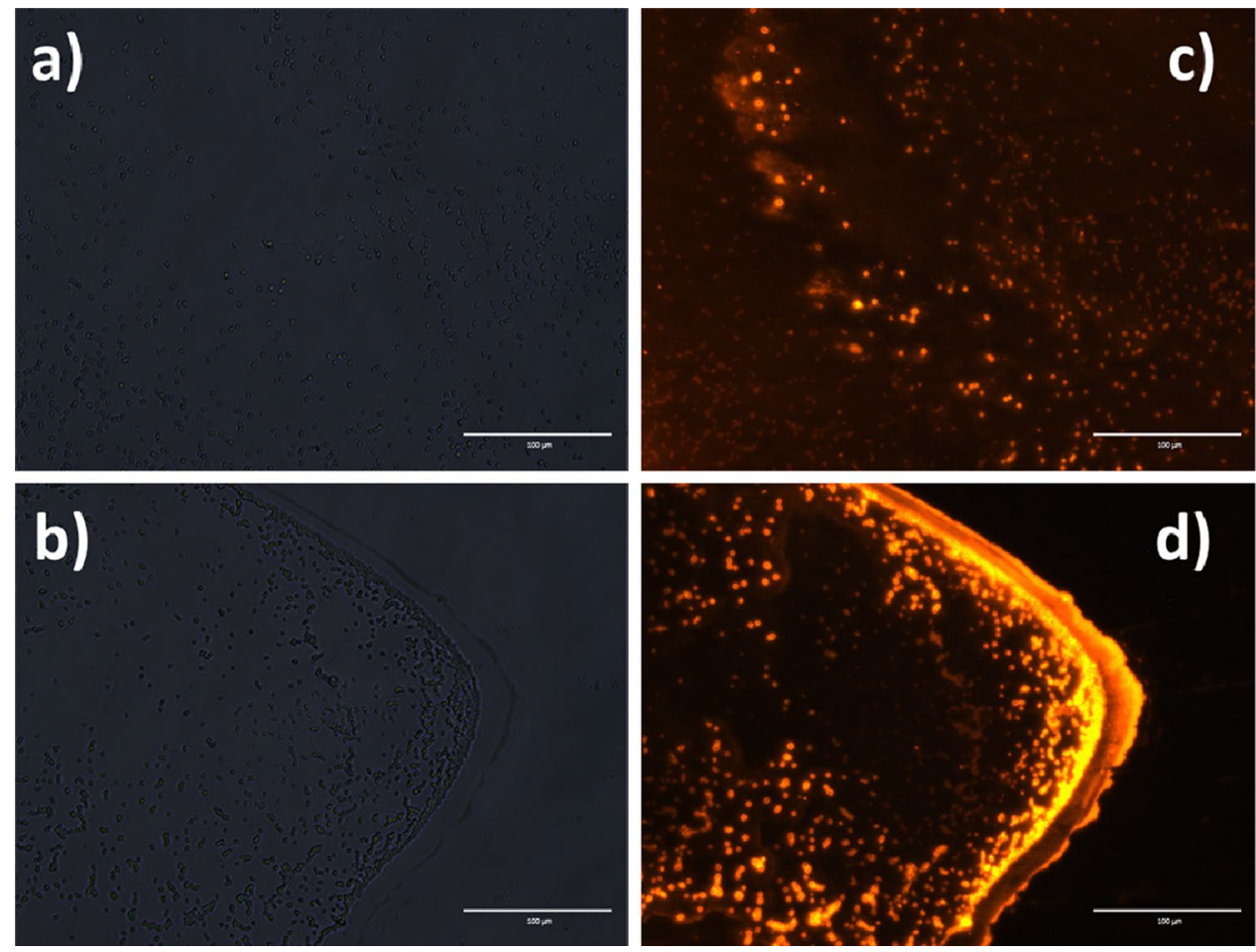

E. coli. From the images, it can be found that intact cytoplasmic membrane was protecting the bacterium. Besides, intercellular communication can also be found, which indicated their exuberant vitality. From the results, it can be observed that the $E$. coli cells incubated with QDs have no significant membrane damage, indicating that the QDs cannot significantly destroy the E. coli microbes. Corresponding EDX spectrum confirms QDs presence by giving the elemental composition of unlabeled and labeled bacterial cells. Thus, E. coli cells were successfully labeled with M-CdTe QDs without any additional target-based functional groups.

The negatively charged surface of E. coli was well protected by its outer membrane and prevents the antibacterial effect of M-CdTe QDs. Thus, the protective layer prevents further penetration into cell wall; the QDs were attached effectively on the cell wall of bacteria. According to the interface model for weakly acidic ionizable groups, the interface is neutral in neutral solution and it becomes negatively charged in a basic one (Trefalt et al. 2016). The additional target-based biomolecules were eliminated to label the microbial cells by maintaining the neutral $\mathrm{pH}$ for an effective attachment of QDs on the cell wall through the charge (electron) transfer mechanism.
This study was able to address novel insight about the clear mechanism of cell labeling by charge transfer-based interface chemistry.

\section{Conclusion}

This study has demonstrated clear insights into solventbased dynamic radius and interface potential effect on the surface of QDs. Based on the solvent physiochemical parameters, the interaction mechanism is elucidated in detail with the help of characterization studies. In vitro cytotoxic and antimicrobial potential of QDs were analyzed to label the animal and bacterial cells to avoid excess dosage that causes the photokilling effect. With prior knowledge in $\mathrm{pH}$ influences on photophysical properties of QDs, the neutral $\mathrm{pH}$ was found to be optimum to label the $E$. coli cells. Interaction of QDs with animal and bacterial cells was confirmed by fluorescence microscopy images. The morphology of bacterial cells and the confirmation of labeling were analyzed by SEM and EDX studies. Charge transfer between QDs and bacteria that avoids endocytosis of cell has helped in biolabeling. These exciting developments will find unseen applications in labeling science 

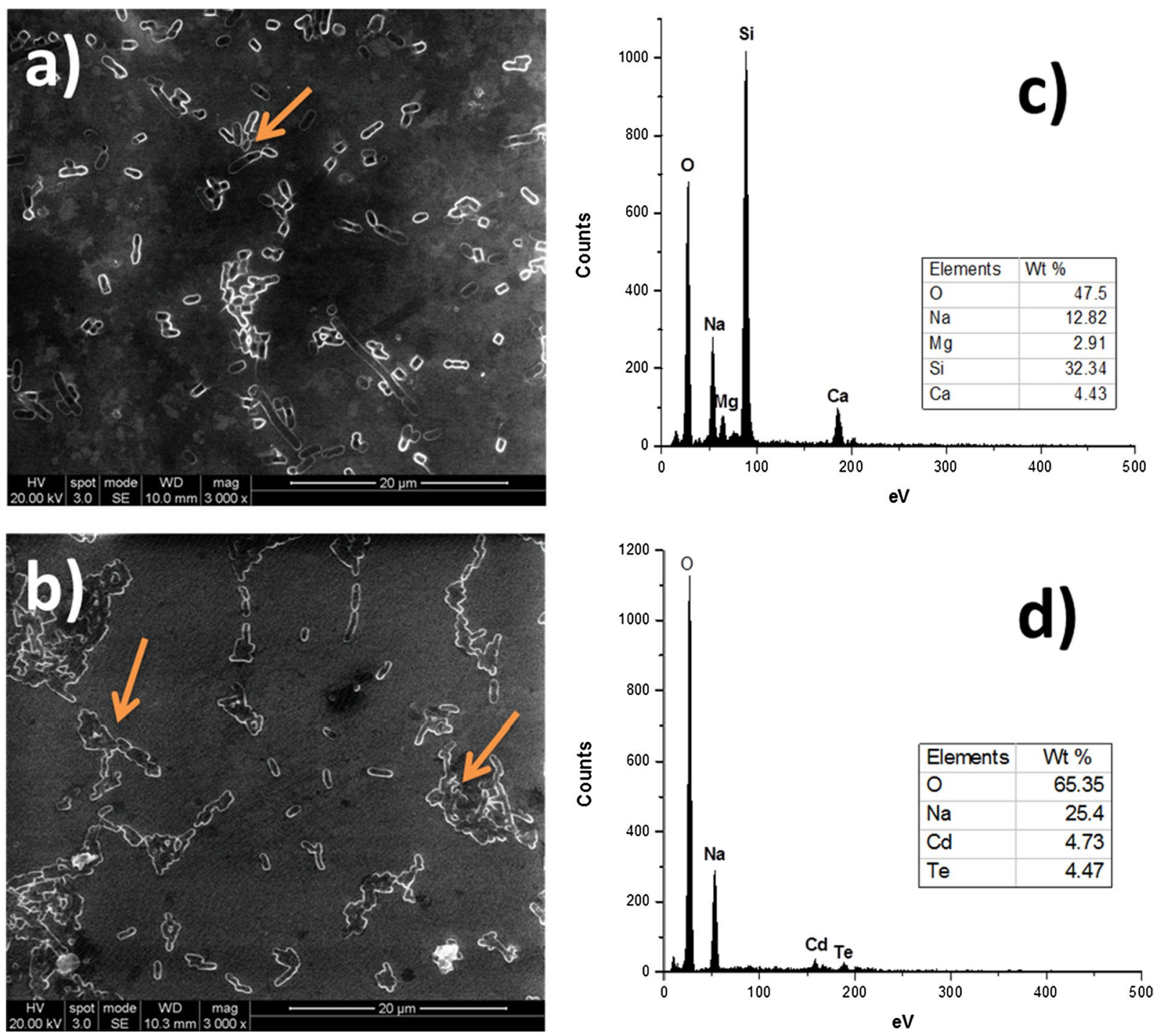

Fig. 10 Biolabeling of bacteria by M-CdTe QDs SEM images. a Control cells (arrow marks indicate non-agglomerated bacterial cells; $\mathbf{b}$ labeled cells (arrow marks indicate the QDs induced agglomeration of bacterial cells), $\mathbf{c}, \mathbf{d}$ corresponding EDX spectrum

by enhancing and complementing QDs as existing arsenal bioprobe.

Acknowledgements The author (S. Kanagasubbulakshmi) would like to thank DRDO for providing fellowship. This work was financially supported by DRDO. The authors also would like to thank Naveen Kumar Kalagatur for toxicity studies.

\section{References}

Arakha M, Saleem M, Mallick BC, Jha S (2015) The effects of interfacial potential on antimicrobial propensity of $\mathrm{ZnO}$ nanoparticle. Sci Rep. https://doi.org/10.1038/srep09578

Boles MA, Ling D, Hyeon T, Talapin DV (2016) The surface science of nanocrystals. Nat Mater 15:141-153. https://doi.org/10.1038/ nmat 4526
Chen J, Song JL, Sun XW et al (2009) An oleic acid-capped CdSe quantum-dot sensitized solar cell. Appl Phys Lett 94:15-18. https ://doi.org/10.1063/1.3117221

DeBlois RW, Bean CP, Wesley RKA (1977) Electrokinetic measurements with submicron particles and pores by the resistive pulse technique. J Colloid Interface Sci 61:323-335. https://doi. org/10.1016/0021-9797(77)90395-2

Du Y, Huang Z, Wu S et al (2018) Preparation of versatile yolk-shell nanoparticles with a precious metal yolk and a microporous polymer shell for high-performance catalysts and antibacterial agents. Polym (UK) 137:195-200. https://doi.org/10.1016/j.polym er.2017.12.069

Ellis JL, Hickstein DD, Schnitzenbaumer KJ et al (2015) Solvents effects on charge transfer from quantum dots. J Am Chem Soc 137:3759-3762. https://doi.org/10.1021/jacs.5b00463

Gao M, Kirstein S, Möhwald H et al (1998) Strongly photoluminescent CdTe nanocrystals by proper surface modification. J Phys Chem B 102:8360-8363. https://doi.org/10.1021/jp9823603 
Gao X, Cui Y, Levenson RM et al (2004) In vivo cancer targeting and imaging with semiconductor quantum dots. Nat Biotechnol 22:969-976. https://doi.org/10.1038/nbt994

Gaponik N, Rogach AL (2010) Thiol-capped CdTe nanocrystals: progress and perspectives of the related research fields. Phys Chem Chem Phys 12:8685. https://doi.org/10.1039/c000916d

Jhonsi MA, Renganathan R (2010) Investigations on the photoinduced interaction of water soluble thioglycolic acid (TGA) capped CdTe quantum dots with certain porphyrins. J Colloid Interface Sci 344:596-602. https://doi.org/10.1016/j.jcis.2010.01.022

Jiang F, Muscat AJ (2013) Solvent-triggered self-assembly of CdTe quantum dots into flat ribbons. J Phys Chem C 117:22069-22078. https://doi.org/10.1021/jp406795t

Kloepfer JA, Mielke RE, Nadeau JL (2005) Uptake of CdSe and CdSe/ $\mathrm{ZnS}$ quantum dots into bacteria via purine-dependent mechanisms uptake of $\mathrm{CdSe}$ and $\mathrm{CdSe} / \mathrm{ZnS}$ quantum dots into bacteria via purine-dependent mechanisms. Appl Environ Microbiol 71:25482557. https://doi.org/10.1128/aem.71.5.2548

Li W, Xie H, Xie Z et al (2004) Exploring the mechanism of competence development in Escherichia coli using quantum dots as fluorescent probes. J Biochem Biophys Methods 58:59-66. https ://doi.org/10.1016/s0165-022x(03)00154-4

Lüpertz R, Wätjen W, Kahl R, Chovolou Y (2010) Dose- and timedependent effects of doxorubicin on cytotoxicity, cell cycle and apoptotic cell death in human colon cancer cells. Toxicology 271:115-121. https://doi.org/10.1016/j.tox.2010.03.012

Michalet X, Gambhir SS, Weiss S (2005) Quantum dots for live cells. Science 307:538-544

Murray CB, Norris D, Bawendi MG (1993) Synthesis and characterization of nearly monodisperse $\mathrm{CdE}(\mathrm{E}=\mathrm{S}, \mathrm{Se}, \mathrm{Te})$ semiconductor nanocrystallites. J Am Chem Soc 115:8706-8715. https://doi. org/10.1021/ja00072a025

Noh M, Kim T, Lee H et al (2010) Fluorescence quenching caused by aggregation of water-soluble CdSe quantum dots. Colloids Surfaces A Physicochem Eng Asp 359:39-44. https://doi. org/10.1016/j.colsurfa.2010.01.059

Oszwałdowski S, Roberts KP, Timerbaev AR (2013) Capillary zone electrophoresis of quantum dots dispersed in mixed micelles: new evidence of the concentration effect. J Chromatogr A 1305:320 327. https://doi.org/10.1016/j.chroma.2013.07.047

Pattekari P, Zheng Z, Zhang X et al (2011) Top-down and bottomup approaches in production of aqueous nanocolloids of low solubility drug paclitaxel. Phys Chem Chem Phys. https://doi. org/10.1039/c0cp02549f

Pyell U (2010) Characterization of nanoparticles by capillary electromigration separation techniques. Electrophoresis 31:814-831. https://doi.org/10.1002/elps.200900555

Schneider R, Wolpert C, Guilloteau H et al (2009) The exposure of bacteria to CdTe-core quantum dots: the importance of surface chemistry on cytotoxicity. Nanotechnology 20:225101. https://doi. org/10.1088/0957-4484/20/22/225101

Sierra MB, Pedroni VI, Buffo FE et al (2016) Colloids and surfaces $\mathrm{B}$ : biointerfaces the use of zeta potential as a tool to study phase transitions in binary phosphatidylcholines mixtures. Colloids Surfaces B Biointerfaces 142:199-206. https://doi.org/10.1016/j. colsurfb.2016.02.061
Smith AM, Duan H, Rhyner MN, Ruan G (2006) www.rsc.org/pccpl Physical Chemistry Chemical Physics A systematic examination of surface coatings on the optical and chemical properties of semiconductor quantum dots w. 3895-3903. https://doi.org/10.1039/ b606572b

Trefalt G, Behrens SH, Borkovec M (2016) Charge regulation in the electrical double layer: ion adsorption and surface interactions. Langmuir 32:380-400. https://doi.org/10.1021/acs.langm uir.5b03611

Uyeda HT, Medintz IL, Jaiswal JK et al (2005) Synthesis of compact multidentate ligands to prepare stable hydrophilic quantum dot fluorophores. J Am Chem Soc 127:3870-3878. https://doi. org/10.1021/ja044031w

Venkataramana M, Chandra Nayaka S, Anand T et al (2014) Zearalenone induced toxicity in SHSY-5Y cells: the role of oxidative stress evidenced by $N$-acetyl cysteine. Food Chem Toxicol 65:335-342. https://doi.org/10.1016/j.fct.2013.12.042

Vergaro V, Scarlino F, Bellomo C et al (2011) Drug-loaded polyelectrolyte microcapsules for sustained targeting of cancer cells. 63:847-864. https://doi.org/10.1016/j.addr.2011.05.007

Wang MY, Yang YY, Heng PWS (2004) Role of solvent in interactions between fatty acids-based formulations and lipids in porcine stratum corneum. J Control Release 94:207-216. https://doi. org/10.1016/j.jconrel.2003.10.016

Wang W, Ding X, Xu Q et al (2016) Colloids and surfaces B: biointerfaces zeta-potential data reliability of gold nanoparticle biomolecular conjugates and its application in sensitive quantification of surface absorbed protein. Colloids Surfaces B Biointerfaces 148:541-548. https://doi.org/10.1016/j.colsurfb.2016.09.021

Wu X, Liu H, Liu J et al (2003) Immunofluorescent labeling of cancer marker Her2 and other cellular targets with semiconductor quantum dots. Nat Biotechnol 21:41-46. https://doi.org/10.1038/ nbt764

Wu Y, Li Q, Deng F et al (2014) Solvent effect on $\zeta$ potential at an aqueous/oil interface in surfactant-free emulsion. Langmuir 30:1926-1931. https://doi.org/10.1021/la403900e

Wuister SF, De Mello Donegá C, Meijerink A (2004) Influence of thiol capping on the exciton luminescence and decay kinetics of CdTe and CdSe quantum dots. J Phys Chem B 108:17393-17397. https ://doi.org/10.1021/jp047078c

Zhang H, Zhou Z, Yang B, Gao M (2003) The influence of carboxyl groups on the photoluminescence of mercaptocarboxylic acidstabilized CdTe nanoparticles. J Phys Chem B 107:8-13. https:// doi.org/10.1021/jp025910c

Zhang C, Ji X, Zhang Y et al (2013) One-pot synthesized aptamerfunctionalized CdTe : Zn 2 + quantum dots for tumor-targeted fluorescence imaging in vitro and in vivo. Anal Chem. https://doi. org/10.1021/ac400606e

Zheng Z, Zhang X, Carbo D et al (2011) NIH public access. Water 26:7679-7681. https://doi.org/10.1021/la101246a

Publisher note Springer Nature remains neutralwith regard to jurisdictional claims in published maps andinstitutional affiliations. 